\title{
Temporal and Motor Transfer in Music Performance
}

\author{
ROSALEE K. MEYER \& CAROLINE PALMER \\ Ohio State University
}

\begin{abstract}
Three transfer-of-learning experiments were conducted to investigate performers' ability to generalize knowledge of specific temporal structure and motor movements from one melody to another. Skilled pianists performed one melody during 10 training trials and another melody during 4 test trials, under speeded performance conditions. In Experiment 1, the meter and/or motor movements (hand and finger assignments) were altered from training to test melodies; in Experiment 2, the rhythm and/ or motor movements were altered; in Experiment 3, the meter and/or rhythm were altered. Differences in total melody duration from training to test were smaller when meter, rhythm, or motor variables were retained across sequences. Furthermore, the same variables of meter, rhythm, and motor movements influenced the tempo of each performance. These findings support distinct temporal and motor representations underlying performance of simple melodies.
\end{abstract}

Received January 20, 2003; accepted July 1, 2003.

$\mathrm{T}$ HE goal of the current study is to determine how temporal structure and motor movements are represented when performers learn new musical pieces. When musicians learn to perform a novel melody, they must learn what events (pitches or chords) to produce, when to produce the events (timing), and how to produce the events (motor movements). Performers can learn musical pieces at many levels of representation that include cognitive and motoric aspects of sequence structure (Chaffin \& Imreh, 2002; Palmer \& Meyer, 2000; Palmer \& van de Sande, 1993). Studies of sequence learning in other domains have focused on effector-specific (motoric) and effector-independent representations (Cohen, Ivry, \& Keele, 1990;

Address correspondence to Caroline Palmer, Dept. of Psychology, McGill University, 1205 Dr. Penfield Ave, Montreal H3A 1B1 Quebec, Canada. (e-mail: caroline.palmer@ mcgill.ca or meyerr@battelle.org)

ISSN: 0730-7829. Send requests for permission to reprint to Rights and Permissions, University of California Press, 2000 Center St., Ste. 303, Berkeley, CA 94704-1223. 
Grafton, Hazeltine, \& Ivry, 1998) but few have addressed how motoric and temporal aspects of sequences are combined in tasks as rich as music performance.

Although the timing of individual elements is an explicit goal of music performance that has been a focus of much study (see Clarke, 1999; Gabrielsson, 1999; Palmer, 1997 for reviews), less is known about how performers encode temporal and motor dimensions when learning musical sequences. Knowledge of when to produce an event may be specified in terms of a set of effector-specific motor movements, or it may be abstract and independent of the necessary movements. Some researchers have argued that representations of timing in sequence production are not effector-specific (MacKay, 1982, 1987; Semjen \& Ivry, 2001). Although support for effector-independent sequence representations has been reported in language production and music performance (MacKay \& Bowman, 1969; Palmer \& Meyer, 2000), these studies did not address temporal aspects of sequence structure. The time at which sequence events should occur must ultimately influence the motor act of producing those events, and it is possible that temporal and motoric levels of representation might interact in learning musical sequences.

We investigate the relationship between two types of temporal structure in music: rhythm and meter, and their relation to motor movements as performers learn musical sequences. Rhythm refers here to the relative durations of sequence events (the pattern of interonset intervals, or IOIs), and meter refers to the hierarchical periodic structure of musical beats. Three experiments are reported that address the following issues in music performance: (1) which temporal and motoric aspects of musical sequences are represented during learning, (2) whether temporal aspects of musical sequences are represented in terms of motor movements, and (3) whether different temporal structures (meter and rhythm) are represented in an independent or interactive manner.

\section{Effector Movements in Learning Musical Sequences}

Some theories of sequence learning propose abstract representations that are independent of the specific effector movements used to generate the sequence (Ivry, 1996; Keele, 1981). For example, handwriting samples produced with different effectors (such as different hands or feet) by the same person tend to be similar, even though different muscles are used for each instance, implying that some representation of handwriting exists independently of the motor movements necessary to execute the action (Bernstein, 1967; Ivry, 1996). Transfer-of-learning tasks have been used to demon- 
strate evidence for effector-independent sequence representations; transfer of learning refers to a change in response on a novel task as a function of experience on a prior task. Transfer of learning across effector movements was demonstrated for keypress sequences that were produced with different arm or finger movements (Cohen et al., 1990; Keele, Cohen, \& Ivry, 1990) and for sentences produced by bilinguals in different languages (MacKay \& Bowman, 1969). These studies and others (see also Grafton et al., 1998) suggest that some encoding during learning occurs at an abstract level that is independent of the particular effectors used to perform the task.

Using a transfer-of-learning paradigm, Palmer and Meyer (2000) tested effector independence in piano performance. Skilled pianists performed a novel melody as quickly as possible during training. At test, pianists performed another musical sequence with either the same or different melodic (pitch order) structure and either the same or different motor (hand and finger) movements. The most transfer in melody duration was observed when the same pitch order was retained from training to test; changes in motor movements did not affect their rate of performance. These results suggested that melodies were learned primarily in terms of their conceptual, rather than motoric aspects. However, novice child pianists performing the same task demonstrated transfer only to sequences with identical motor and melodic dimensions; more experienced child pianists showed transfer on both the motor and the melodic dimensions. These findings suggested that less skilled performers represented musical sequences in terms of both melodic (nonmotoric) and motoric aspects, and skilled performers represented them primarily in terms of melodic (nonmotoric) aspects.

These transfer-of-learning studies did not investigate whether temporal aspects of the sequences were learned; for example, Palmer and Meyer's (2000) notated musical sequences contained isochronous pitches. The representation of temporal structure in piano performance, for example, may be in terms of abstract codes or in terms of specified arrival times of fingers on keys. The transfer-of-learning experiments presented here address whether representations of temporal structure are abstract or motoric in music performance. In the next section, we address the role of two temporal structures in music performance: rhythm and meter.

\section{Temporal Structures in Musical Sequence Learning}

Rhythm, defined here as the pattern of IOIs in a melodic sequence, is an important psychological dimension of temporal structure not only in mu-

sic but in many types of sequences (Martin, 1972; Rosenbaum, 1991; Shaffer, 
1982). Some have suggested that rhythm is represented independently of motor movements in sequence learning (Schmidt, 1975). According to proposals of generalized motor programs (Schmidt, 1975; Vorberg \& Wing, 1996), centrally stored patterns specify the relative timing of commands in abstract (nonmotoric) form, which is input to a motor system that produces a temporally structured output. Thus, these proposals suggest that the representation of a sequence's temporal structure is not effector-specific.

Meter, another important dimension of temporal structure in music, is a hierarchical structure of alternating strong and weak beats or pulses (the temporal units of a musical sequence) that form accents (Cooper \& Meyer, 1960; Lerdahl \& Jackendoff, 1983). The beats that compose each hierarchical level are (approximately) equally spaced apart in time; beats occurring at larger periods define higher metrical levels, and beats occurring at smaller periods define lower metrical levels. Shaffer, Clarke, and Todd (1985) proposed that performers' representations of musical meter are abstract (nonmotoric), whereas representations of rhythm are motoric. Following Wing and Kristofferson's (1973) two-stage model of timekeeping, they proposed a central timekeeper that operates at one hierarchical level of metrical structure, and motor procedures that specify movement patterns of rhythms operating at lower levels (Shaffer, 1982; Shaffer et al., 1985). The first two experiments reported here extend this prediction to test whether representations of rhythm are motoric (effector-specific) and of meter are nonmotoric (effector-independent).

We also address whether performers represent the different temporal structures (meter and rhythm) of music independently. Some music theorists have suggested that meter and rhythm do not function independently of each other (Cooper \& Meyer, 1960; Hasty, 1997); meter may function as a rule system that generates appropriate rhythms (Longuet-Higgins \& Lee, 1982; Shaffer et al., 1985). Some perceptual theories also predict an interaction between meter and rhythm (Longuet-Higgins \& Lee, 1982; Parncutt, 1994; Povel \& Essens, 1985). In Parncutt's (1994) model, the perception of meter is a function of the tempo and rhythm in a sequence. In Povel and Essens' (1985) model, listeners find the best regular (metrical) internal clock for a rhythmic pattern; the clock that conflicts the least with the rhythm provides the best fit and is perceived as the beat. In LonguetHiggins and Lee's (1982) model, listeners alter or confirm their perception of the meter for a rhythmically varying sequence as each new IOI occurs. Meter and rhythm interact because the perception of a meter affects subsequent rhythm perception, and the perception of the rhythmic pattern can influence the perceived meter. Although there is less known about relationships among rhythm and meter in performance, some evidence from expressive timing in performance suggests that rhythmic relationships dominate metrical relationships when the two conflict (Drake \& Palmer, 1993). The final experiment presented here investigates the relationship between meter and rhythm in performers' transfer of learning. 
We contrast performers' representations of temporal and motor structure in three experiments, using a transfer-of-learning paradigm. In each experiment, skilled pianists were trained on one novel melody and then performed another melody at test, under speeded conditions. Specific transfer, or transfer that can be attributed to specific relationships between two tasks (Underwood, 1983), is commonly measured in performance under speeded conditions by the difference in task duration from the end of training to test. We measured musical transfer similarly here, in terms of the difference in pianists' total melody duration from the end of training to test. In Experiment 1, the meter and/or the required motor (hand and finger) movements for the musical sequences were altered from training to test to address whether meter is an effectorspecific component of sequence representations. In Experiment 2, the rhythm and/or the motor movements for the musical sequences were altered from training to test to study whether rhythm is an abstract or motoric component of sequence representations. In Experiment 3, the meter and/or the rhythm of musical sequences were altered from training to test in order to identify whether these temporal structures are represented independently.

To address the role of temporal and motor structures independent of other melodic variables, the melodies contained the same serial ordering of pitches across training and test in each experiment. The serial order of movements and of pitches are often correlated in piano performance. For example, keypresses by the thumb followed by the little finger of the right hand produce an ascending melodic contour of pitches, and keypresses generated by the little finger followed by the thumb produce a descending contour of pitches. The musical sequences used in Experiment 1, shown in Figure 1, demonstrate how the serial ordering of pitches was kept constant while the hand and finger movements were altered: numbers under each musical example indicate the instructed finger movements for the hand assigned ( $1=$ thumb, $5=$ little finger). The independent manipulation of meter, rhythm, and motor movements with serial ordering of pitches held constant allows us to evaluate the temporal and motor components of sequence learning independence of possible pitch-ordering factors. Meter, rhythm, and motor movements are independently manipulated in pairs across three experiments (as opposed to their simultaneous manipulation in a single experiment, to avoid the use of stimuli that are too artificial).

\section{Experiment 1: Meter and Motor Movements}

Experiment 1 investigated the role of meter and motor movements in performers' representations for melodies. Pianists performed melodies during training and test that contained the same serial ordering of pitches; the hand and finger movements and the notated metrical structure were either the same or different from training to test. Pianists performed as quickly as 


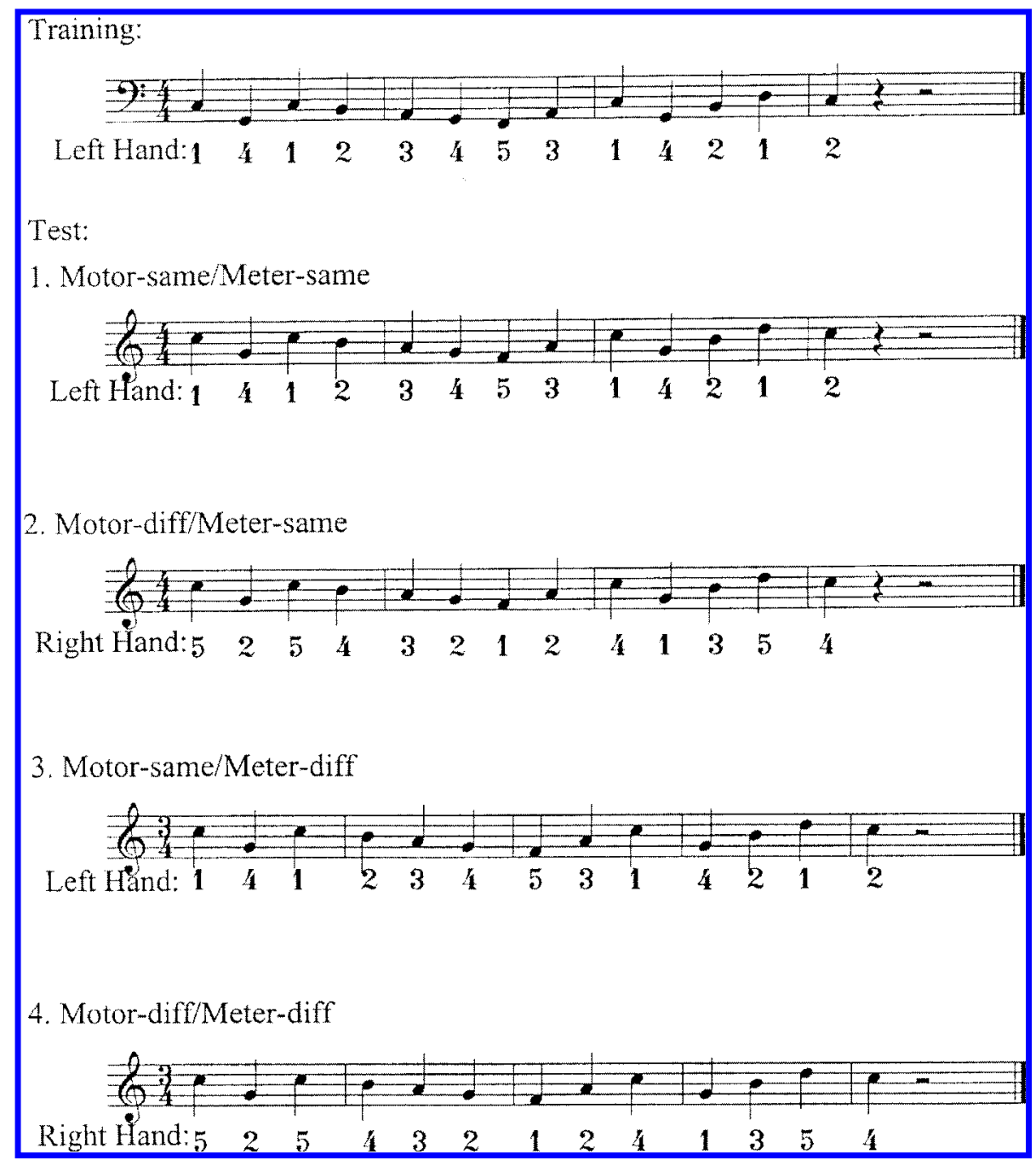

Fig. 1. One training melody and four test melodies used in Experiment 1.

possible, and transfer of learning was measured by the difference in total melody duration between the end of training and test. Each pianist participated in all conditions, and "Filler" tunes (unrelated to the experimental melodies) were performed by pianists between conditions to control for carryover effects such as memory for earlier sequences.

\section{METHOD}

\section{Participants}

Sixteen adult pianists (mean age $=25$ years, range $=18-48$ years) were recruited from the Columbus, Ohio music community. The pianists had 13.3 years of piano instruction on 
average (range $=8-18$ years) and a mean of 17.9 years of performing experience (range $=8-$ 40 years) and all reported being right-handed. Pianists received either partial course credit or a nominal fee for their participation.

\section{Materials}

Four sets of eight isochronous melodies, each containing 13 quarter-note pitches (with no rhythmic variation), were created for use in Experiment 1. Although the melodies differed in the hand and finger movements required to produce them, they were all designed to be ergonomically simple (e.g., none contained any repeating fingers or large pitch intervals that required repositioning of the hand). In a pilot study, eight skilled pianists, who did not participate in Experiment 1, performed and rated 28 melodic sequences on how difficult each melody was to perform with the notated hand and meter on a scale from 1 (very hard) to 7 (very easy). The ratings revealed a subset of sequences with no significant rating differences that were included in Experiment 1; these were in different keys (half were major and half were minor), and were relatively easy to perform. Codings of ergonomic complexity (Parncutt, Sloboda, Clarke, Raekallio, \& Desain, 1997) confirmed that the melodies were equivalently easy. Thus, the sequences were designed to minimize any differences due to reading difficulty, ergonomic requirements, or fits of the same pitch sequence to different meters.

Each set of eight sequences contained all combinations of clef (treble and bass), hand and finger assignments (right and left), and meter (3/4 and 4/4) for a single melody. The serial order of pitches was preserved in all melodies within each set, but the meter and/or finger movements were altered between sequences across hands. Therefore, changing the motor movements changed both the hand and finger assignments, whereas the serial order of pitches remained the same. The clef in which the melodies were notated was altered from training to test so that the test sequence was never visually identical to the training sequence. Figure 1 shows one training stimulus and examples of the four possible test sequences. The remaining three stimuli for the set shown in Figure 1 are the same as Stimuli 2, 3 , and 4 in the figure, but notated in the bass clef.

Four "filler" tunes, 13-note isochronous sequences for two hands based on arpeggiated chords, were also composed. Fillers were composed in different meters (two were in 3/4 and two were in 4/4 meter) and in different keys and were also different from the keys of the test stimuli. Each filler required use of all five fingers in both hands, to reduce any motor carryover effects between preceding and following sequences.

\section{Equipment}

Participants performed on a computer-monitored Boesendorfer 290SE Imperial Grand piano (1.25-ms resolution); the same equipment was used in all experiments. Computer software determined keypress onsets and offsets and identified pitch errors by comparing the performance with the pitch information in the notated musical score.

\section{Design and Procedure}

Independent variables of motor movements (same or different hand/finger relationships from training to test) and meter (same or different meter from training to test) were manipulated in a within-subjects design. The meter variable is referred to as Meter-same or Meter-diff (different), and the motor variable is referred to as Motor-same or Motor-diff (different). The four conditions were ordered according to a Latin square design, with a melody from a different set assigned to each condition for each pianist. Each stimulus set appeared equally often in each condition across pianists. The following variables were balanced across subjects and conditions: whether the musical melody was performed by the right or left hand, whether the melody was notated in bass or treble clef, and which stimulus set was assigned to each condition. Thus, each participant received one training melody from each set, as determined by the Latin square design that counterbalanced the remaining variables. Hand and finger assignments for melodies were counterbalanced across both conditions and performers. 
Participants completed the following procedure for each condition: They first performed a filler sequence four times as quickly as possible. Then, they began a training session by performing a training melody slowly until no errors were made (to ensure that any errors were not due to perceptual or reading errors); all participants performed the training melody without any errors within three pretraining trials. During the training stage, participants performed the same training melody 10 times as quickly as possible. Next, they performed a test melody four times as quickly as possible in the test stage. Examples of a training melody and possible test melodies for each condition are shown in Figure 1. The same procedure was repeated for the remaining three conditions, using a new filler and new stimulus set for each condition. The dependent variables were total melody duration (onset of last produced tone minus onset of first tone) and pitch error rate per trial.

\section{RESULTS}

\section{Melody Durations}

Figure 2 shows the mean melody durations of training and test trials for Experiment 1 after outliers computed across subjects and conditions were removed (12 of 896 trials were outside three standard deviations from the mean). An analysis of variance (ANOVA) on sequence durations for only the training trials by trial (10) and which transfer condition the training trials preceded (4) indicated a main effect of training trial, $F(9,135)=$ $22.07, p<.01$. There were no training differences across the four conditions, indicating no group differences among pianists at baseline (practice).

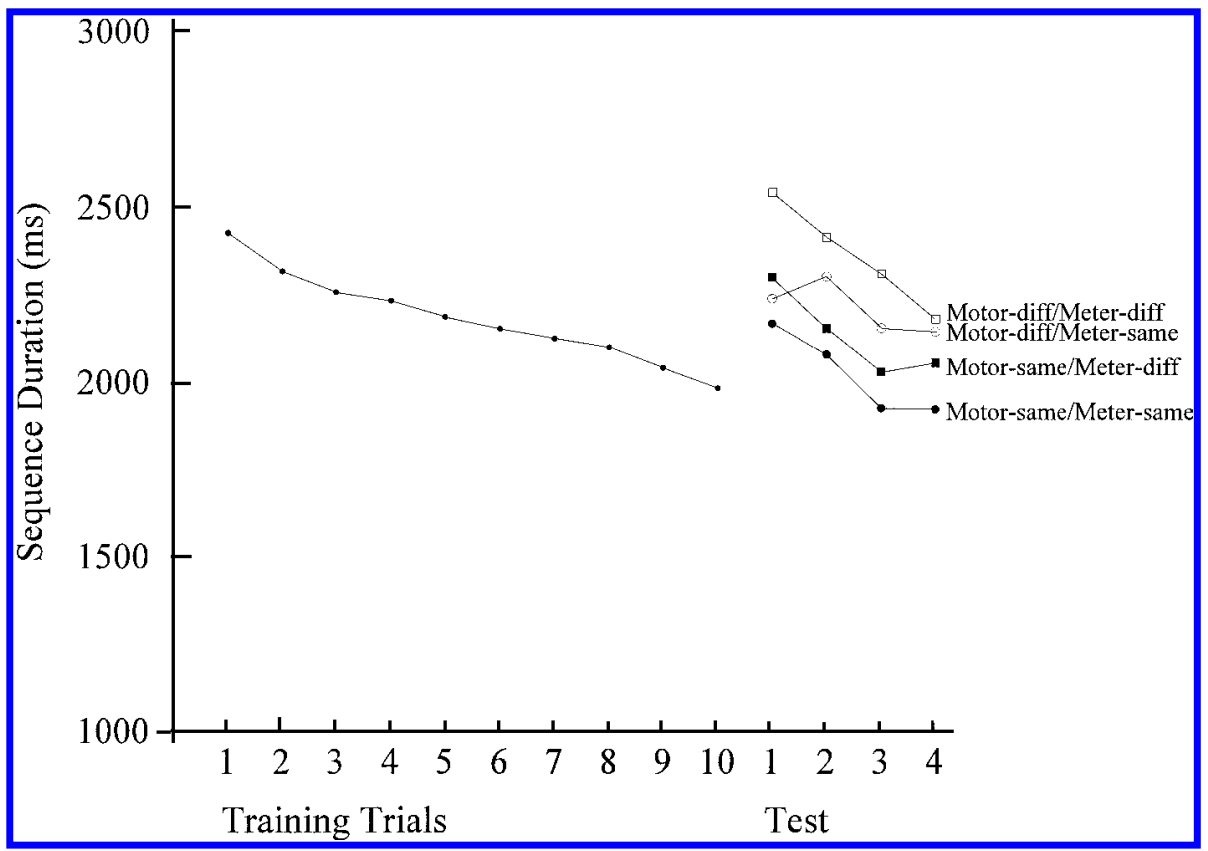

Fig. 2. Mean melody durations of training and test trials in Experiment 1. 
The total sequence durations of the training trials were examined for evidence of improvement following a power law. The correlation between training trial number and sequence duration averaged over subjects and performances indicated a significant negative relationship on both a linear scale $(r=-.94, p<.01)$ and a $\log -\log$ scale $(r=-.99, p<.01)$. A significance test between dependent $r$ 's (Cohen \& Cohen, 1983) indicated that the log relationship was significantly larger than the linear relationship, $t(7)=$ $2.72, p<.05$. Log-scale correlations within individual performers were significant for all 16 pianists (range $=-.77$ to $-.99, p<.01$ ). As shown in Figure 2, improvement in melody duration was greatest in the first few training trials.

Transfer of learning was measured as the difference between the total melody duration of the last training trial and the mean melody duration of the four test trials. An ANOVA on the difference scores indicated a significant main effect of motor condition, $F(1,15)=11.34, p<.01$, and a significant main effect of meter condition, $F(1,15)=7.39, p<.05$, and no interaction. Planned comparisons on the difference scores from training to test suggested that changes in motor movements had greater effects on transfer than did changes in meter. There were significant differences between the Motor-same/Meter-same and Motor-diff/Meter-same conditions $(p<$ $.05)$, and between the Motor-same/Meter-diff and Motor-diff/Meter-diff conditions $(p<.05)$. There were no significant differences between the Motor-same/Meter-same and Motor-same/Meter-diff conditions, or between the Motor-diff/Meter-same and Motor-diff/Meter-diff conditions. Perfect transfer, defined as no significant change in sequence duration from the final training trial to the mean of test trials, was observed for the Motorsame/Meter-same condition only.

Differences in melody duration within the test trials indicated significant main effects of condition, $F(3,45)=5.27, p<.01$, and trial, $F(3,45)=$ $8.58, p<.01$, but no interaction. The difference in melody duration from the last training trial (Trial 10) to the mean duration of the first two test trials indicated a significant main effect of both motor condition, $F(1,15)$ $=8.72, p<.05$, and meter condition, $F(1,15)=5.26, p<.05$. The same analysis on the difference between the last training trial and the mean duration of the last two test trials indicated a significant effect of motor condition only, $F(1,15)=13.90, p<.01$. Overall, these results confirm that performers were able to adjust to a change in meter more quickly than they were able to adjust to a change in hand and finger movements. There were no effects of filler items or order of conditions on the results.

To evaluate whether the smaller effects of meter on melody durations resulted from a failure to notice a change in notated meter for the test melodies, hammer velocities (a measure of loudness) in the performances were examined for evidence of metrical sensitivity; previous studies indi- 
cate that events in strong metrical positions are performed louder than events in weak metrical positions (Sloboda, 1983; Drake \& Palmer, 1993). Events on beats 1 and 3 in 4/4 meter and on beat 1 in 3/4 meter were coded as strong metrical positions, and the remaining events were coded as weak metrical positions. Events in strong metrical positions were performed significantly louder than events in weak metrical positions in the test melodies, $F(1,15)=13.78, p<.01$, for melodies notated in both meters. Thus, the weaker transfer of meter was not due to a failure to interpret the sequences as having a particular metrical structure.

\section{Errors}

The mean of pitch error rates (number of pitch errors divided by number of notated pitches) was .016, indicating that pitch performance was close to perfect in training and test trials. Duration errors were defined as events whose IOIs were outside three standard deviations from the mean IOI of the isochronous events. The mean of duration error rates (number of duration errors divided by number of IOIs) was .012, indicating that duration performance was close to perfect in training and test trials. Neither pitch nor duration error rates differed across trials or conditions.

\section{DISCUSSION}

Transfer-of-learning effects indicated that meter and motor movements were retained in performers' representations of musical melodies; performances were slower when either the meter or the hand and finger assignments of the musical sequences changed from training to test, with larger transfer effects for motor movements. Motor movements had independent effects from those of meter; changing one variable did not affect transfer of learning for the other variable. In contrast, Palmer and Meyer (2000) found that changes in motor movements had no transfer effects in skilled pianists' performance of musical melodies. Temporal structures such as meter, which were not investigated in Palmer and Meyer (2000), may increase the relative importance of motor movements in sequence learning. Rhythm, or the relative timing of event durations, is another temporal structure that may influence the importance of motor movements. The relationship between

motor movements and rhythm in sequence representations is investigated in the next experiment.

\section{Experiment 2: Rhythm and Motor Movements}

In Experiment 2, the same transfer-of-learning paradigm was used to evaluate rhythm and motor components of performers' representations of 
melodies. In addition to hand and finger assignments, the rhythm (pattern of IOIs) was manipulated from training melody to test melody. Again, the serial ordering of pitch events was kept constant across training and test melodies.

\section{METHOD}

\section{Participants}

Sixteen adult pianists (mean age $=21$ years, range $=16-45$ years) were recruited from the Columbus, Ohio music community. The pianists had 10.1 years of piano instruction on average (range $=7-14$ years) and a mean of 13.9 years of performing experience $($ range $=8-$ 40 years). Fourteen pianists reported being right-handed, and two reported being left-handed. Two of the pianists from Experiment 1 participated in Experiment 2. Pianists received either partial course credit or a nominal fee for their participation.

\section{Materials}

Four sets of eight melodies, each containing 19 pitches, were created for use in Experiment 2 . The required hand and finger movements were designed to be ergonomically equivalent and easy, as in Experiment 1. The melodies were chosen from a pilot study in which eight skilled pianists, who did not participate in Experiment 2, performed and rated 32 melodies on how difficult each melody was to perform with the notated hand and rhythm. A subset of the sequences that yielded no significant rating differences was used in the experiment; the sequences were in different keys (half were major and half were minor) and were relatively easy to perform. Each set of eight sequences contained all combinations of clef (treble, bass), hand and fingers (right and left), and rhythms (two for each set). Figure 3 contains an example of one training melody and four test melodies from one set. Each rhythmic pattern, composed from a different combination of two different duration values (e.g., quarter notes and eighth notes), repeated in each measure of a melody. The two rhythms within each set were related such that one notated duration was replaced with the other notated duration (quarter notes were replaced with two eighth notes, and two eighth notes were replaced by a quarter note). This manipulation allowed the rhythm to change from sequences at training to test, while keeping the total number of unique durations and the total number of beats the same. Examples of the motor and rhythmic manipulations in training and test melodies are shown in Figure 3. Four new filler sequences were composed for Experiment 2 according to the same criteria as in Experiment 1.

\section{Design and Procedure}

The design and procedure was the same as in Experiment 1, except that the variables manipulated from training to test included motor movements (same or different hand and finger relationships, called Motor-same and Motor-diff, respectively) and rhythm (same or different rhythm, called Rhythm-same and Rhythm-diff, respectively). A different filler item was performed 4 times before each condition, and sequences from a new stimulus set were used for each of the four conditions.

\section{RESULTS}

\section{Melody Durations}

Figure 4 shows the mean total melody durations of training and test trials for Experiment 2 after outliers (durations outside three standard deviations from the mean) were removed (less than $1 \%$ of all trials). An 
Training:

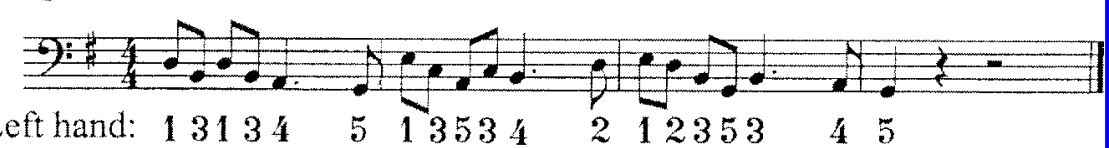

Left hand: $13134 \quad 513534 \quad 212353 \quad 45$

Test:

1. Rhythm-same/Motor-same

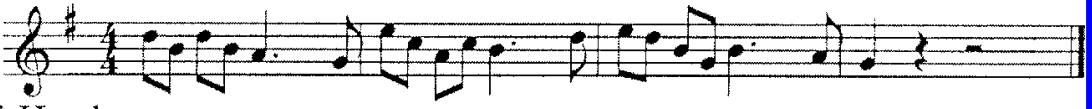

Left Hand: $13134 \quad 51353421235345$

2. Rhythm-diff/Motor-same

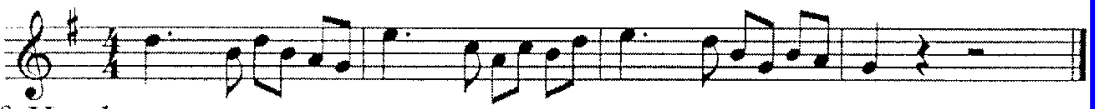

Left Hand: $1 \quad 313451353421235345$

3. Rhythm-same/Motor-diff

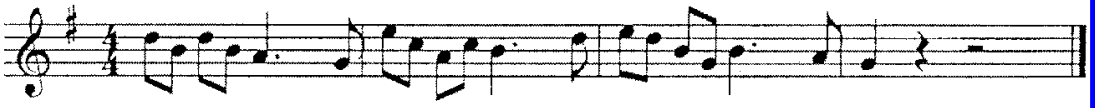

Right hand: $53532 \quad 153132454213 \quad 21$

4. Rhythm-diff/Motor-diff

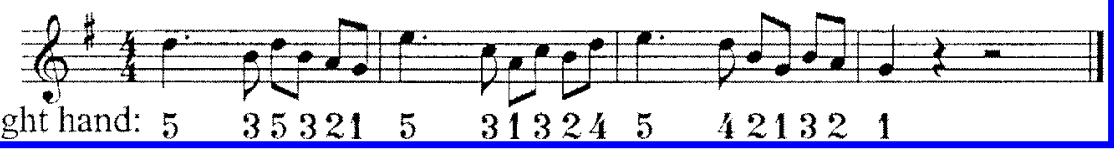

Fig. 3. One training melody and four test melodies used in Experiment 2.

ANOVA on melody durations for the training trials indicated a main effect of training trial, $F(9,135)=28.87, p<.01$, and no differences across conditions. Training trials were examined for evidence of a power law relationship between practice and sequence duration. The correlation between training trial number and sequence duration averaged over subjects and performances indicated a significant negative relationship on both a linear scale $(r=-.94, p<.01)$ and a log-log scale $(r=-.98, p<.01)$. A significance test between dependent $r$ 's indicated that the log relationship was significantly larger than the linear relationship $(t(7)=2.68, p<.05)$. Correlations on the log-log scale were significant for all 16 pianists $(r=-.78$ to 


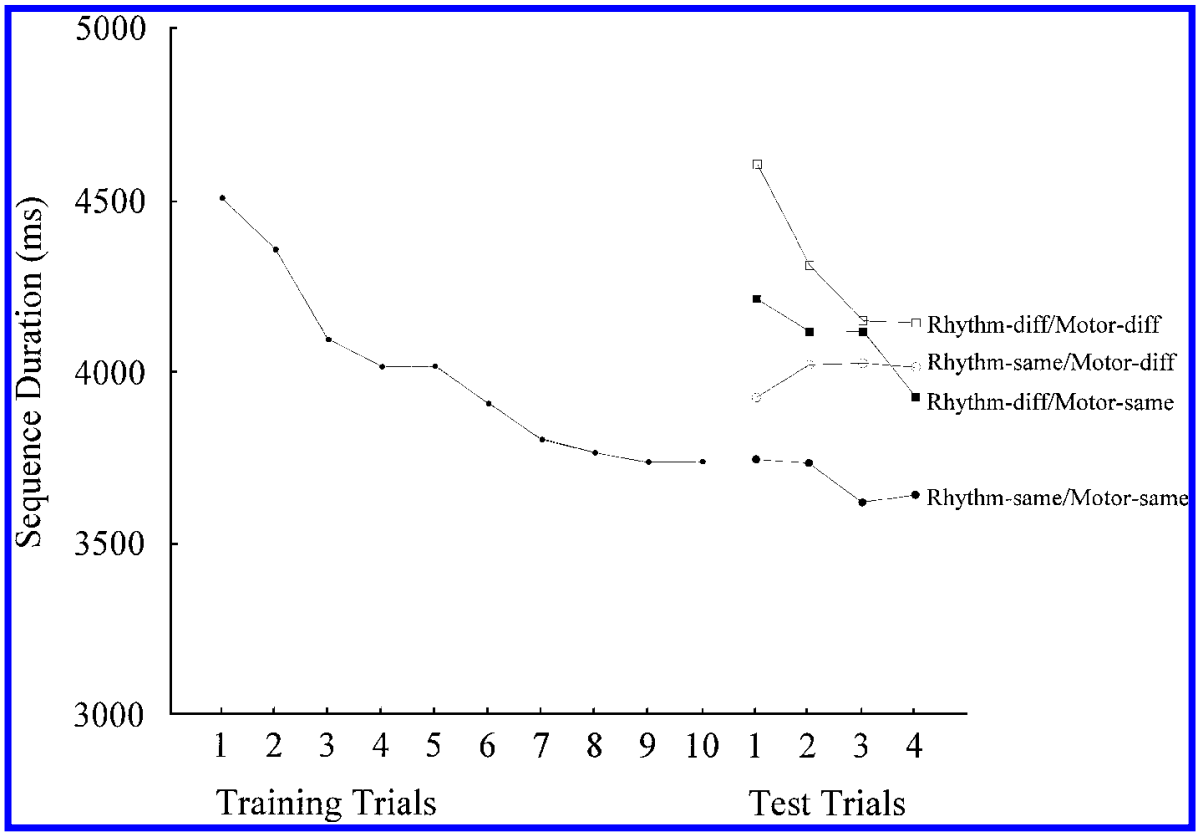

Fig. 4. Mean melody durations of training and test trials in Experiment 2.

$-.99, p<.01)$. Most improvement in melody duration was seen early in practice.

Analyses on the difference between the total melody duration of the last training trial and the mean of the test trials indicated significant main effects of motor condition, $F(1,15)=14.39, p<.01$, and rhythm condition, $F(1,15)=7.99, p<.05$, and no interaction. Planned comparisons indicated significant differences between the Rhythm-diff/Motor-same and Rhythm-same/Motor-same conditions $(p<.01)$ and between the Rhythmsame/Motor-diff and Rhythm-same/Motor-same conditions $(p<.05)$. Perfect transfer (no significant change in sequence duration from the final training trial to the mean of test trials) was observed for the Rhythm-same/ Motor-same condition only.

Analyses on melody durations within the test trials also indicated significant differences among the four conditions, $F(3,45)=5.43, p<.01$, and among the four trials, $F(3,45)=4.21, p<.01$. There was also a significant interaction between conditions and trials, $F(9,135)=2.5, p<.05$; as shown in Figure 4, the Rhythm-same/Motor-diff condition did not asymptote during test in the same manner as the other three conditions. Differences between the last training trial and the first two test trials indicated significant main effects of motor condition, $F(1,15)=14.6, p<.01$, and rhythm condition, $F(1,15)=11.9, p<.01$, and no interactions. The same analysis on the differences from training to the last two test trials revealed 
the same motor effects, $F(1,15)=11.20, p<.01$, and rhythm effects, $F(1$, $15)=5.20, p<.05$, and no interaction. These results confirm that both rhythm and motor movements facilitated transfer from one melody to another. There were no significant effects of filler stimuli or order of conditions.

\section{Errors}

Pitch and duration errors were examined for transfer effects. The mean pitch error rate was .014 , indicating that pitch performance was close to perfect in training and test trials. Duration errors were identified for the rhythmically varying stimuli as follows (see also Drake \& Palmer, 2000): The mean IOI of each duration category (eighth-note, quarter-note, and dotted quarter-note) was computed for each performance. IOIs more than halfway between the mean of the correct duration category and the next larger or smaller duration category were considered duration errors. This method of identifying duration errors allowed some flexibility in the performed tempo without unduly increasing the number of events coded as duration errors. The mean duration error rate was .011 , indicating that duration performance was close to perfect in training and test trials. Neither pitch nor duration error rates changed significantly over trials or conditions, due to the very low error rate overall.

\section{DISCUSSION}

Experiment 2 confirmed that rhythmic structure and motor movements are retained in performers' representations of musical sequences; pianists' performances were slower when either the rhythm or the hand and finger assignments were changed from training to test, with larger effects of rhythm on transfer. Transfer of rhythm was observed even under speeded conditions of performance, extending previous findings of rhythm transfer in a simpler task (Summers, 1975). Effects of motor movements were independent from rhythm in the transfer task; changing one variable did not affect amount of transfer on the other variable. The failure to find an interaction between temporal structure and movement in either Experiments 1 or 2 suggests independent temporal and motor components in performers' representations of simple melodies.

\section{Experiment 3: Meter and Rhythm}

Experiment 3 investigated whether the temporal structures of meter and rhythm are represented independently in performances of novel melodies. Pianists performed musical sequences in the same transfer-of-learning para- 
digm; the meter and rhythm of musical sequences were kept the same or changed from training to test trials, while the motor movements and serial order of the pitches remained the same (all melodies were performed with the right hand).

\section{METHOD}

\section{Participants}

Sixteen adult pianists ( mean age $=20$ years, range $=18-25$ years) were recruited from the Columbus, Ohio music community. The pianists had 10.3 years of piano instruction on average (range $=7-15$ years) and a mean of 12.1 years of performing experience (range $=9$ 17 years). All pianists reported being right-handed and had not participated in the previous experiments. Pianists received either partial course credit or a nominal fee for their participation.

\section{Materials}

Four sets of eight novel musical sequences, each containing 19 pitches, were created for use in Experiment 3 and were tested for their performance difficulty. In a pilot study, eight skilled pianists who did not participate in Experiment 3 performed and rated 32 melodies on how difficult each melody was to perform with the notated meter and rhythm, on a scale from 1 (very hard) to 7 (very easy). The ratings revealed melody sequences that did not differ significantly; these were in different keys (half were major and half were minor) and were relatively easy to perform.

Each set of eight melodies contained all combinations of clef (treble and bass), meter (3/4 and 4/4), and rhythm (two duration patterns). Figure 5 contains one training and four test melodies from one stimulus set. All melodies were performed with the right-hand fingers (motor movements were not manipulated in Experiment 3). The rhythmic patterns were composed of eighth notes, quarter notes, and dotted quarter notes, and were 12 beats long (three measures in 4/4 meter or four measures in 3/4 meter). To increase the metrical ambiguity of each sequence, different rhythmic patterns were used from one measure within each melody to the next; this helped prevent the rhythmic pattern from fitting one meter better than another. Thus, the rhythmic patterns were more complex in Experiment 3 than in Experiment 2. Four new filler sequences were composed for Experiment 3 according to the same criteria as in the previous experiments.

\section{Design and Procedure}

Experiment 3 used the same design and procedure as in the other experiments, but with independent variables of meter (same or different meter from training to test, called Metersame and Meter-diff, respectively) and rhythm (same or different rhythm from training to test, called Rhythm-same and Rhythm-diff, respectively). An example of one training melody and its test sequences is shown in Figure 5. Melodies from a new stimulus set were used for each condition, and a different filler was performed four times before each condition.

\section{RESULTS}

\section{Melody Durations}

Figure 6 shows the mean total melody durations of training and test trials for Experiment 3 after outliers were removed (less than $1 \%$ of trials were outside three standard deviations from the mean). An ANOVA on 


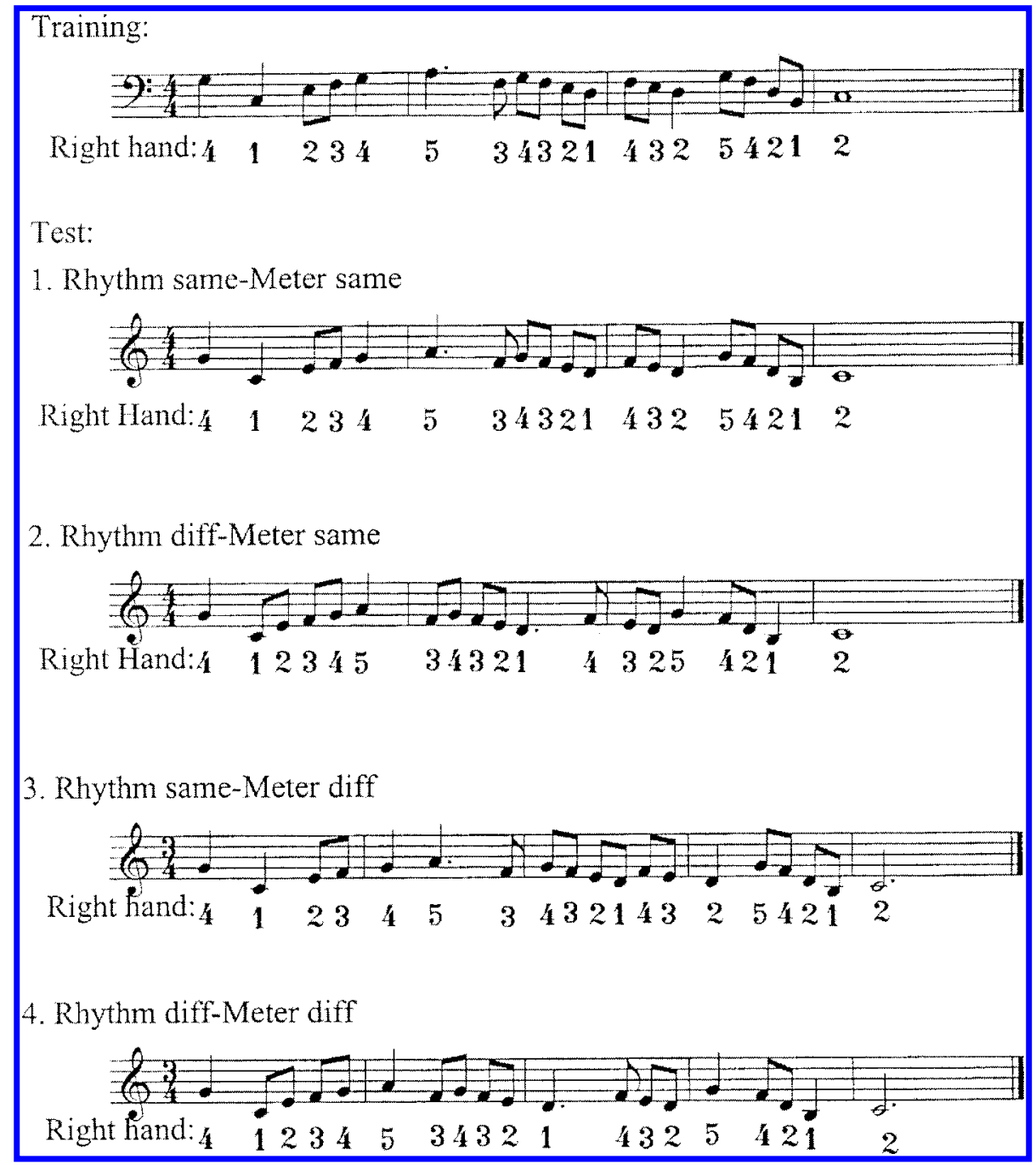

Fig. 5. One training melody and four test melodies used in Experiment 3.

sequence durations for only the training trials indicated a main effect of training trial, $F(9,135)=55.48, p<.01$, and no differences across meter or rhythm conditions. The correlation between training trial number and melody duration averaged over subjects and performances indicated a significant negative relationship on both a linear scale $(r=-.95, p<.01)$ and a $\log$-log scale $(r=-.997, p<.01)$. A significance test between dependent $r$ 's indicated that the log relationship was significantly larger than the linear relationship $(t(7)=2.69, p<.05)$. Individual correlations on the log-log scale were significant for all performers $(r=-.81$ to $-.99, p<.01)$. Total melody durations improved the most early in practice for these rhythmically complex sequences. 


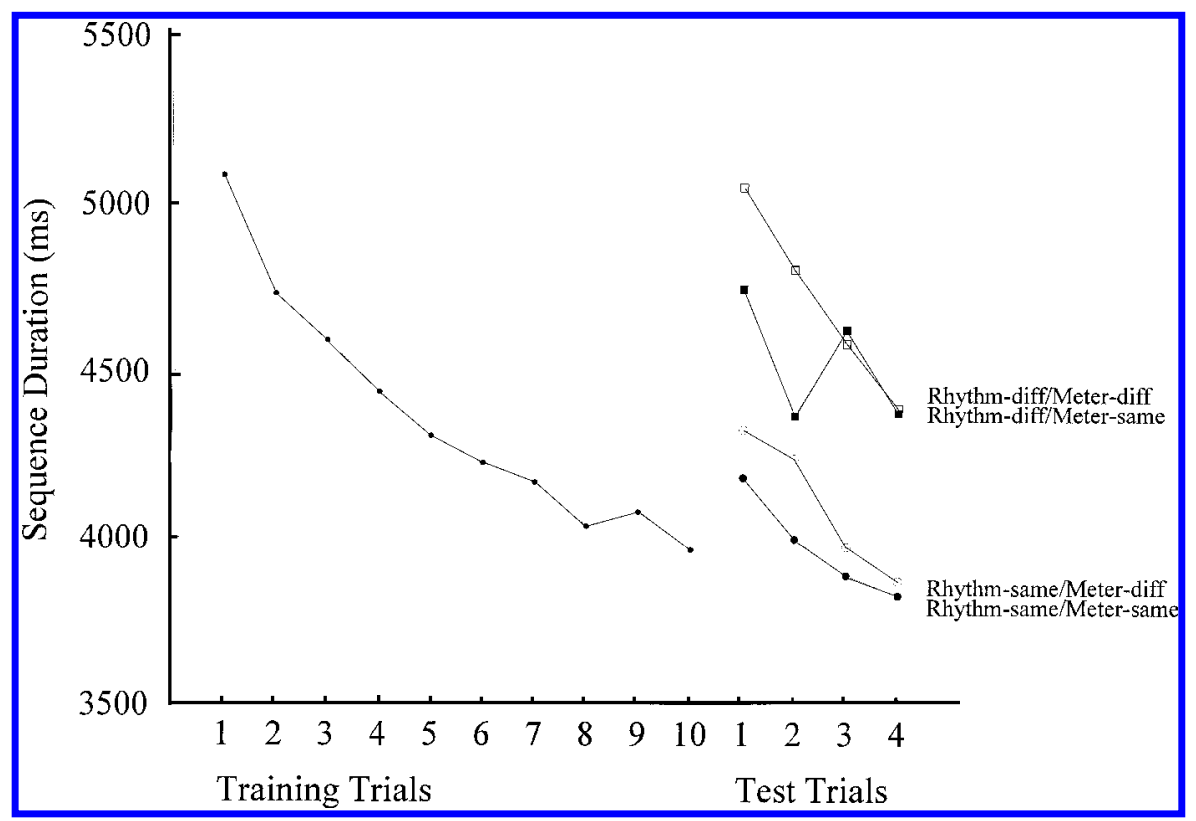

Fig. 6. Mean melody durations of training and test trials in Experiment 3.

Analyses of the difference in total melody duration between the last training trial and the mean of the test trials indicated a significant main effect of rhythm condition, $F(1,15)=21.14, p<.01$, and no effects of meter condition. Planned comparisons among all pairs of conditions also suggested a larger effect of rhythm than meter: There were significant differences between the Rhythm-same/Meter-same and Rhythm-diff/Meter-same conditions $(p<.01)$ and between the Rhythm-diff/Meter-diff and Rhythm-same/ Meter-diff conditions $(p<.01)$. The Rhythm-same/Meter-same and Rhythmsame/Meter-diff conditions did not differ significantly; nor did the Rhythmdiff/Meter-diff and Rhythm-diff/Meter-same conditions. Perfect transfer (no significant change in total duration from the final training trial to the mean of test trials) was observed for both the Rhythm-same/Meter-diff and Rhythm-same/Meter-same conditions.

Examination of the melody durations within the test trials indicated significant main effects of the four conditions, $F(3,45)=8.34, p<.01$, and the four trials, $F(3,45)=17.51, p<.01$, and no interaction. Differences in melody durations between the last training trial and the first two test trials indicated significant main effects of meter, $F(1,15)=6.93, p<.05$, and rhythm, $F(1,15)=16.26, p<.01$. The same analysis on the difference in melody durations between the last training trial and the last two test trials indicated a significant effect of rhythm only, $F(1,15)=20.67, p<.01$. Similar to the findings in Experiment 1, the transfer-of-meter findings suggest that performers were able to learn to change the meter more quickly 
(within 4 test trials) than they could learn to change the rhythm. There were no effects of filler stimuli or order of conditions on melody durations.

\section{Errors}

The mean pitch error rate (.009) and duration error rate (.01), defined as in Experiment 2, indicated that performance was close to perfect in training and test trials. There were no differences in error rates across conditions.

\section{Comparisons Across Experiments}

To evaluate the relative contributions of rhythm, meter, and motor components to melody representations simultaneously, we compared the performances from all experiments. Each melody was coded in terms of three complexity measures: motor complexity (number of unique finger transitions), rhythmic complexity (number of unique duration transitions), and metrical complexity (number of measures). Motor complexity was coded as number of unique finger transitions between successive sequence events in each sequence (range $=10-13$ ). Motor complexity refers here to a cognitive measure of memory for the finger pattern, rather than ergonomic complexity, which was controlled across the sequences. For example, a tone produced with the thumb (1) followed by the third finger (3) at the beginning of a sequence is one unique motor transition, but the same 1-to-3 finger transition occurring later in the same sequence is not unique. Rhythmic complexity was coded as number of unique durational transitions between successive sequence events in each metrical position, ranging from 3 to 15 . Rhythmic transitions were coded relative to metrical position because the rhythms were constructed in Experiment 2 to repeat across metrical positions. For example, a quarter note at the beginning of the first measure followed by an eighth note is a unique rhythmic transition, but the same rhythmic transition at the beginning of the next measure in the same melody is not unique. Metrical complexity was coded as the number of metrical groups (measures) in each melody, as indicated by notated vertical barlines in each sequence, ranging from 4 to 5 . As an example of the coding, the training stimulus in Figure 5 (Experiment 3 ) had a motor complexity of 13 , a rhythmic complexity of 13 , and a metrical complexity of 4 , whereas the last transfer stimulus in Figure 5 had a motor complexity of 13, a rhythmic complexity of 9 , and a metrical complexity of 5 .

Effects of motor, rhythmic, and metrical complexity were examined in terms of the mean IOIs for each performance, in order to adjust for length of melodies across the three experiments. Simple correlations across the complexity variables for all training and test performances from all experi- 
ments indicated that mean IOI correlated positively with rhythmic complexity, $r=.45, p<.01$, and with metrical complexity, $r=.33, p<.01$. The correlation with motor complexity was small and negative, $r=-.05$. A multiple regression analysis, predicting the mean IOI of each performance from the three complexity measures was significant, $R=.63, p<.01$, and the standardized regression coefficients for metrical and rhythmic complexity were significant and positive. Performance was slower on average for melodies that contained more rhythmic complexity (number of events was controlled) and more metrical units. The same multiple regression analysis was significant for training trials, $R=.63, p<.01$, and for test trials, $R$ $=.65, p<.01$, with significant standardized regression coefficients for both metrical and rhythmic complexity $(p<.05)$. The mean IOI for each performance correlated significantly with metrical complexity (training: $r=.33$, test: $r=.34$ ) and with rhythmic complexity (training: $r=.45$, test: $r=.46$, $p<.01)$. Correlations with motor complexity were not significant. Thus, the rate at which pianists performed the melodies across the three experiments reflected the same underlying variables across training and test: rhythmic complexity followed by metrical complexity contributed most to overall speed of performance, and motor complexity (in terms of unique finger transitions) contributed least.

\section{DISCUSSION}

Pianists' transfer of learning from one melody to another indicated greater effects of rhythm than meter in representations of melodies; performances were slower when the rhythm of the musical sequences changed from training to test, and only slower in initial test trials when the meter changed. A new meter may have been easier to learn for a given melody than a new rhythm because the rhythm changed in each measure (whereas the meter stayed consistent). Comparisons across experiments confirmed that the rate at which performers produced individual melodies were indeed influenced more by the rhythmic complexity. The same temporal and motor variables affected individual performances across experiments as affected transfer between simple melodies: rhythmic complexity contributed most and motor complexity contributed least to overall rate of performance.

\section{General Discussion}

The meter, rhythm, and motor movements that pianists learned for one musical sequence aided subsequent performance of another sequence, as demonstrated in a transfer-of-learning task. No interactions were observed among the temporal (meter and rhythm) and motor structures. In addition, 
the metrical and rhythmic complexity of a melody were predictive of the rate at which pianists could perform the melody. These results suggest that (1) redundancy in temporal structure facilitated speeded performance more than redundancy in motor movements, and (2) motor features play a smaller role in transfer of knowledge across melodies, at least for these moderately skilled pianists. Overall, the findings are consistent with the general view that representations of timing in sequence production are not effector specific (MacKay, 1982, 1987; Semjen \& Ivry, 2001) and they extend previous support for effector-independent sequence representations (MacKay \& Bowman, 1969; Palmer \& Meyer, 2000) to temporal aspects of sequence structure. Also consistent is the view that performers learn musical sequences in terms of multiple levels of representation. We review those levels of representation next.

\section{MOTOR TRANSFER IN MUSIC PERFORMANCE}

Performers' representations for musical sequences showed some motor transfer; performances of test melodies were faster when the hand and finger movements were the same at training than when they differed. This finding contrasts with previous studies that did not find a motoric component in transfer of learning, including transfer findings for reaction times to patterns of lights (Cohen et al., 1990) and transfer findings for bilingual speakers' sentence durations (MacKay \& Bowman, 1969). Palmer and Meyer (2000) also found motor independence for skilled pianists' melody representations in a task comparable to the current study. Why would motor independence exist for representations of some musical sequences and not for others? The melodies used in the current studies varied in rhythmic and metrical structures; perhaps temporal variations within a sequence increase the overall difficulty of memory for motor sequences. Planning a series of motor movements whose durations vary may engage a more complex motor program than that required for isochronous sequences, for which an invariant rate might be captured in a single parameter value (Schmidt, 1975).

Another possibility is the greater motor demands on performance in the current melodies; the musical sequences required finger movements that were less redundant than those used by Palmer and Meyer. Eighty-eight percent of the finger transitions in the melodies of Experiment 1 (with isochronous event durations, comparable to those of Palmer \& Meyer, 2000) were unique, compared with $59 \%$ of the finger transitions in Palmer and Meyer's (2000) sequences. Thus, the less redundant motor sequences used in the current studies may have accounted for larger motor effects in sequence learning. The finding that melody representations were effector independent only when temporal or motor demands were low is consistent with findings that novice child performers' representations of melodies are more effector specific in nature than those of skilled adults (Palmer \& Meyer, 
2000). The amount of motor transfer in music performance may be sensitive to the motoric and temporal complexity of the task.

\section{TEMPORAL TRANSFER IN MUSIC PERFORMANCE}

Melodies were performed more rapidly when they retained metrical or rhythmic relationships from previously performed melodies. Several researchers have suggested that meter serves an organizing role in music performance (Palmer \& Pfordresher, in press; Shaffer et al., 1985). Shaffer et al. (1985) suggest that meter functions as a timekeeper to control the timing of a music performance; Meyer and Palmer (1996) found evidence that performers' metrical interpretations influenced the timing of IOIs at different metrical levels. These findings suggest that the hierarchical structure of meter influences the early acquisition (learning) as well as timing of events in music performance. The novel finding of rhythmic transfer across musical sequences is consistent with proposals that IOI patterns are part of motor programs (Schmidt, 1975; Vorberg \& Wing, 1996). Both simple rhythms (Experiment 2) and more complex rhythms (Experiment 3) were performed faster when a previously performed melody shared the same rhythm; the rhythmic pattern was repeated more often within each sequence in Experiment 2, whereas the rhythmic pattern did not repeat in the sequences in Experiment 3 (in order to promote metrical ambiguity). In contrast to Shaffer's proposal that musical rhythms are represented in terms of motor (effector-specific) procedures (Shaffer, 1982; Shaffer et al., 1985), the experiments reported here suggest that representations of rhythm were nonmotoric (effector independent).

The same variables of temporal structure and motor movements that affected transfer of learning across melodies were also predictive of the tempo at which pianists could perform each melody. The more metrically or rhythmically complex a melody was, the slower the melody was performed. The rhythmic complexity contributed most to performance tempo, similar to the transfer findings across experiments that indicated largest effects of rhythm. Metrical complexity also affected tempo; the mean IOI increased linearly with the number of metrical units in the melody, similar to effects of utterance length on speech timing (Sternberg, Knoll, Monsell, \& Wright, 1988); speakers who produced lists of words under speeded instructions showed a linear increase in the mean word duration as the number of words in the list increased. Our findings of increased IOI with number of metrical units (measures) suggest similarly that the number of planned units influences the ability to perform quickly.

One question that arises is whether the findings from a speeded performance task generalize to the more customary unspeeded music performance. Although the speeded performance conditions were unusual for pianists, the improvement they showed in performance rates during learning, which 
followed a power function, were typical of learning rates found for many other skill acquisition tasks (Ivry, 1996; Newell \& Rosenbloom, 1981); sequence durations decreased more rapidly at the beginning than at the end of practice. Furthermore, performers were able to produce rhythmically varying sequences as quickly as possible in Experiments 2 and 3; they were able to maintain the different event durations (as evidenced by low duration error rates) despite the speeded task instructions. Finally, the rate of learning during training fit a power law function for melodies with and without rhythmic variation across experiments. Thus, performers showed similar rates of learning under speeded instructions as previously found for music produced at normal (unspeeded) rates (Drake \& Palmer, 2000) and for production tasks in other domains (Ivry, 1996; Newell \& Rosenbloom, 1981). These results suggest that similar cognitive processes operate for speeded musical tasks as for other sequence production tasks.

In sum, meter, rhythm, and motor movements differentially influenced pianists' ability to transfer knowledge among musical sequences; rhythmic and metrical aspects of melodies influenced transfer independently of motor movements; and the different temporal structures had independent effects on the amount of transfer. The relationship between timing and movement has a long history in musical and psychological theory: in music, as an analogy between physical motion and psychological motion in pitch and time (cf. Clarke, 1999), and in psychology, as the consequence of intrinsic dynamics (Kelso, Holt, Rubin \& Kugler, 1981) or central timekeepers (Semjen \& Ivry, 2001). These views allow for multiple levels of representation that include effector-specific and effector-independent representations. Our findings extend the effector-independent representations view to complex musical sequences that contain rhythmic and metrical variation. Although pitch structure was held constant in these studies, these findings point to a multileveled representation in performance, at least for simple melodies in which pitch order does not vary. How temporal structure, motor movements, and pitch order combine in sequence learning requires further study. ${ }^{1}$

\section{References}

Bernstein, N. (1967). The co-ordination and regulation of movements. New York: Pergamon. $\rightarrow$ Chaffin, R., \& Imreh, G. (2002). Practicing perfection: Piano performance as expert memory. Psychological Science, 13, 342-349.

1. This research was supported by NIMH grant R01-45764 to the second author. Portions of this project fulfilled the requirements for the first author's doctoral dissertation. The authors thank Grant Baldwin, Rich Jagacinski, Neal Johnson, Mari Reiss Jones, Stephen McAdams, Peter Q. Pfordresher, and three anonymous reviewers for comments, and Steven Finney for helpful discussions. 
Clarke, E. F. (1999). Rhythm and timing in music. In D. Deutsch (Ed.), The psychology of music (2nd ed., pp. 473-500). New York: Academic Press.

Cohen, J., \& Cohen, P. (1983). Applied multiple regression/correlation analysis for the behavioral sciences (2nd ed.). Hillsdale, NJ: Erlbaum.

Cohen, A., Ivry, R. I., \& Keele, S. W. (1990). Attention and structure in sequence learning. Journal of Experimental Psychology: Learning, Memory, and Cognition, 16, 17-30.

Cooper, G., \& Meyer, L. B. (1960). The rhythmic structure of music. Chicago: The University of Chicago Press.

Drake, C., \& Palmer, C. (1993). Accent structures in music performance. Music Perception, 10, 343-378.

Drake, C., \& Palmer, C. (2000). Skill acquisition in music performance: Relations between planning and temporal control. Cognition, 72, 1-33.

Gabrielsson, A. (1999). The performance of music. In D. Deutsch (Ed.), The psychology of music (2nd ed., pp. 501-602). New York: Academic Press.

Grafton, S. T., Hazeltine, E., \& Ivry, R. I. (1998). Abstract and effector-specific representations of motor sequences identified with PET. The Journal of Neuroscience, 18, 9420 9428.

Hasty, C. F. (1997). Meter as rhythm. NY: Oxford University Press.

Ivry, R. I. (1996). Representational issues in motor learning: Phenomena and theory. In H. Heuer \& S. Keele (Eds.), Handbook of perception and action (Vol. 2, pp. 263-330). London: Academic Press.

Keele, S. W. (1981). Behavioral analysis of motor control. In V. Brooks (Ed.), Handbook of physiology, vol. 2: Motor control (pp. 1391-1414). Bethesda, MD: American Physiological Society.

Keele, S. W., Cohen, A., \& Ivry, R. I. (1990). Motor programs: Concepts and issues. In Jeannerod, M. (Ed.), Attention and performance XIII: motor representation and control (pp. 77-110). Hillsdale, NJ: Lawrence Erlbaum.

Kelso, J. A. S., Holt, K. G., Rubin, P., \& Kugler, P. N. (1981). Patterns of human interlimb coordination emerge from the properties of non-linear, limit cycle oscillatory processes: Theory and data. Journal of Motor Behavior, 13, 226-261.

Lerdahl, F., \& Jackendoff, R. (1983). A generative theory of tonal music. Cambridge, MA: MIT Press.

Longuet-Higgins, H. C., \& Lee, C. S. (1982). The perception of musical rhythms. Perception, 11, 115-128.

MacKay, D. G. (1982). The problems of flexibility, fluency, and speed-accuracy trade-off in skilled behavior. Psychological Review, 89, 483-506.

MacKay, D. G. (1987). The organization of perception and action: A theory for language and other cognitive skills. New York: Springer-Verlag.

MacKay, D. G., \& Bowman, R. W. (1969). On producing the meaning in sentences. American Journal of Psychology, 82, 23-39.

Martin, J. G. (1972). Rhythmic (hierarchical) versus serial structure in speech and other behavior. Psychological Review, 79, 487-509.

Meyer, R. K., \& Palmer, C. (1996). Production rate and tactus effects in the temporal control of music performance. Abstracts of the Psychonomic Society, 1, 43.

Newell, A., \& Rosenbloom, P. S. (1981). Mechanisms of skill acquisition and the law of practice. In J. R. Anderson (Ed.), Cognitive skills and their acquisition (pp. 1-55). Hillsdale, NJ: Erlbaum.

Palmer, C. (1997). Music performance. Annual Review of Psychology, 48, 115-138.

Palmer, C., \& Meyer, R. K. (2000). Conceptual and motor learning in music performance. Psychological Science, 11, 63-68.

Palmer, C., \& Pfordresher, P. Q. (in press). Incremental planning in sequence production. Psychological Review.

Palmer, C., \& van de Sande, C. (1993). Units of knowledge in music performance. Journal of Experimental Psychology: Learning, Memory, \& Cognition, 19, 457-470. 
Parncutt, R. (1994). A perceptual model of pulse salience and metrical accent in musical rhythms. Music Perception, 11, 409-464.

Parncutt, R., Sloboda, J. A., Clarke, E. F., Raekallio, M., \& Desain, P. (1997). An ergonomic model of keyboard fingering for melodic fragments. Music Perception, 14, 341-382.

Povel, D.-J., \& Essens, P. (1985). Perception of temporal patterns. Music Perception, 2, 411-440.

Rosenbaum, D. A. (1991). Human motor control. San Diego, CA: Academic Press.

Schmidt, R. A. (1975). A schema theory of discrete motor skill learning. Psychological Review, 82, 225-260.

Semjen, A., \& Ivry, R. I. (2001). The coupled oscillator model of between-hand coordination in alternate-hand tapping: A reappraisal. Journal of Experimental Psychology: $\mathrm{Hu}$ man Perception and Performance, 27, 251-265.

Shaffer, L. H. (1982). Rhythm and timing in skill. Psychological Review, 89, 109-122.

Shaffer, L. H., Clarke, E. F., \& Todd, N. P. (1985). Metre and rhythm in piano playing. Cognition, 20, 61-77.

Sloboda, J. A. (1983). The communication of musical metre in piano performance. Quarterly Journal of Experimental Psychology, 35, 377-396.

Sternberg, S., Knoll, R. L., Monsell, S., \& Wright, C. E. (1988). Motor programs and hierarchical organization in the control of rapid speech. Phonetica, 45, 175-197.

Summers, J. J. (1975). The role of timing in motor program representation. Journal of Motor Behavior, 7, 229-241.

Underwood, B. J. (1983). Transfer and interference. In Attributes of human memory (pp. 180-209). Dallas, TX: Scott Foresman.

Vorberg, D., \& Wing, A. M. (1996). Modeling variability and dependence in timing. In H. Heuer \& S. Keele (Eds.), Handbook of perception and action (Vol. 2, pp. 181-262). London: Academic Press.

Wing, A. M., \& Kristofferson, A. B. (1973). Response delays in the timing of discrete motor responses. Perception \& Psychophysics, 14, 5-12. 